\title{
4-(3,4-Dichlorophenyl)-6-(Furan-2-yl)Pyrimidine-2-Thiol as Building Block in Heterocyclic Synthesis
}

\author{
M. A. EL-Hashash ${ }^{\mathrm{a}}$, Y. A. EL-Badry ${ }^{\star b}$, and J. M. Morsy ${ }^{\mathrm{c}}$
}

${ }^{a}$ Chemistry department, Faculty of Science, Ain Shams University, 11566 Abbasseya, Cairo, Egypt.

${ }^{b}$ Organic Chemistry lab., Faculty of Specific Education, Ain Shams University, 11566 Abbasseya, Cairo, Egypt.

yaser75moemen@yahoo.com

${ }^{\mathrm{c}}$ Synthetic Org. Chemistry Lab., Faculty of Education, Ain Shams University, Cairo, Egypt.

\section{ABSTRACT}

Pyrimidine-2-thiol (1) has been synthesized via one-pot, three component synthesis. Its behavior towards nitrogen nucleophiles like hydrazine hydrate, ethylamine, piperidine, and 2-aminobenzoic acid has been studied. A speculation to explain the activities of the thioamide-iminothiol equilibrium based on their thermodynamic and kinetic control under the experimental conditions were investigated. The hydrazinopyrimidine 2 and aminocarbamoyl derivative 8 were used as key starting materials for synthesis of some interesting nitrogen bridgehead compounds 10-13, hydrazones 14-16, and some mixed and non-mixed heterocyclic systems 17-19 respectively.

\section{Indexing terms/Keywords}

Hydrazinylpyrimidine; Oxadiazolo-pyrimidine; Pyrazolo-pyrimidine; Thioxopyrimidine; Triazolo-pyrimidine.

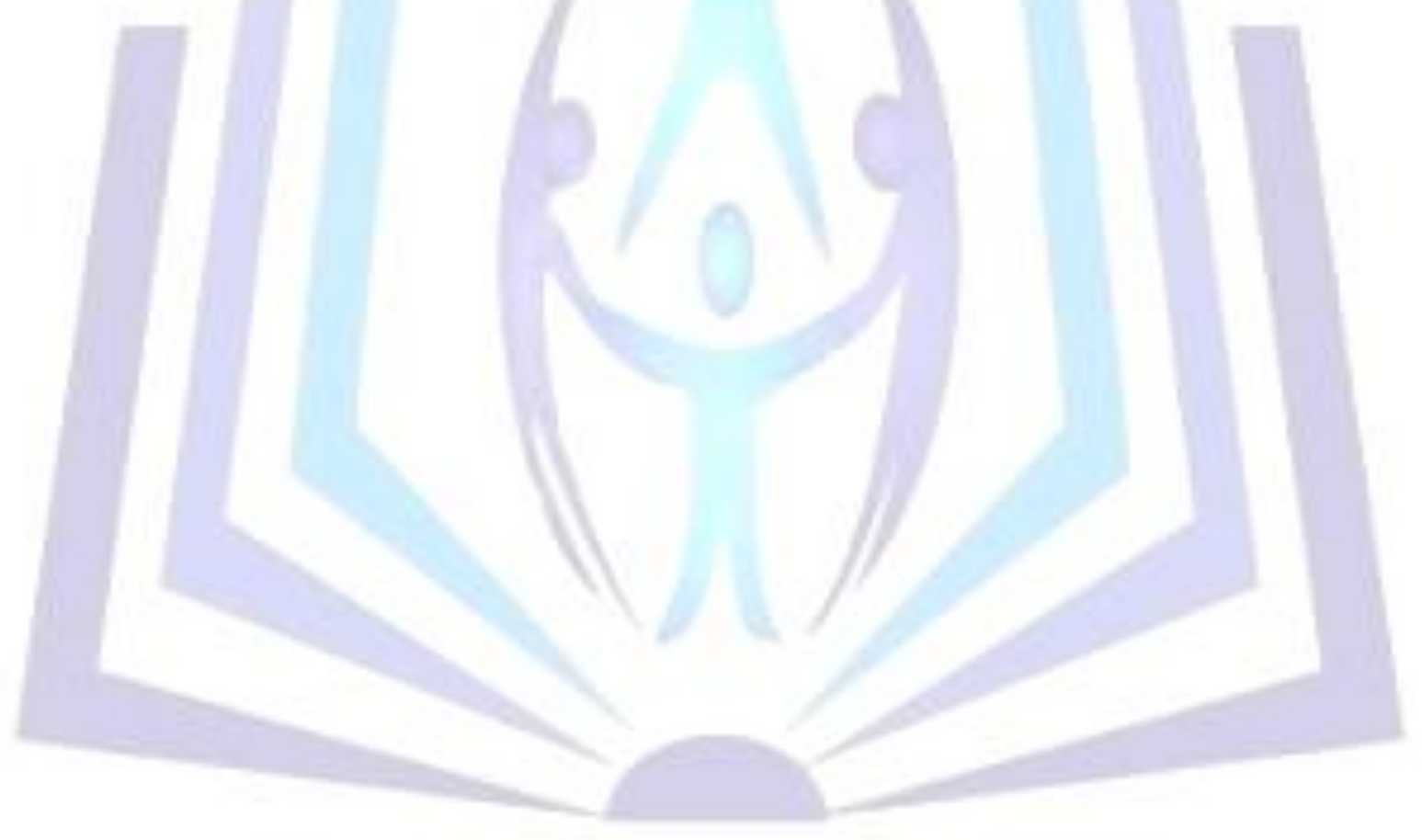

\section{Council for Innovative Research}

Peer Review Research Publishing System

\section{Journal: Journal of Advances in Chemistry}

Vol. 5, No. 2

\author{
editor@cirworld.com
}

www.cirworld.com, member.cirworld.com 


\section{INTRODUCTION}

In 1893 Italian Chemist Pietro Biginelli reported on the acid-catalysed cyclocondensation reaction of ethyl acetoacetate, benzaldehyde, and urea [1]. The reaction was carried out simply by heating a mixture of the three components dissolved in $\mathrm{EtOH}$ with a catalytic amont of $\mathrm{HCl}$ at refluxing temperature. The product of this novel one-pot, three-component synthesis that precipitated on cooling of the reaction mixture was identified correctly by Biginelli as 3,4-dihydropyrimidin$2(1 H)$-one. The synethetic potential of this new heterocycle synthesis (now known as Biginelli reaction) remained unexplored for quite some time.

In the 1970s and 1980s interest slowly increased, and the scope of the original cyclocondensation reaction was gradually extended by variation of all three builiding blocks, allowing access to a large number of multifunctionallized dihydropyrimidines $[2,3]$. Since that late 1980 s, a tremendous increase in activity has again occurred, as evidenced by the growing number of publications and patents on that subject $[3,4]$. This is mainly due to the fact that the multifunctionallized dihydropyrimidine "DHPMs" represents a heterocyclic system of remarkable pharmacological efficiency. In the past decades, a broad range of biological effects including antiviral, antitumor, antibacterial, and anti-inflammatory activities has been ascribed to these partialy reduced pyrimidine derivatives [5-13]. More recently, DHPMs have emerged as for example, orally active antihypertensive agents [14-18].

A very recent highlight in this context has been identified structurally rather simple DHPM monastrol as a mitotic Kinesin motor protein inhibitor and potential new lead for the development of anticancer drug [19]. Appropriately functionalized DHPMs have emerged as potent calcium channel blockers [20-21]. A part from synthetic DHPM derivatives, several marine natural products with interesting biological activities containing the dihydropyrimidine-5-carboxylate core have recently been isolated [22]. Most notably among these are the batzelladine alkaloids $A$ and $B$ which inhibit the binding of HIV envelope protein gp-120 to human CD4 cells and therefore, are potential new leads for AIDS therapy [23].

Herein, we report the synthesis of a thioxopyrimidine derivative $\mathbf{1}$ and its uses as key starting material for synthesis of some interesting heterocycles.

\section{EXPERIMENTAL}

\section{General procedure}

Reagents and solvents were used as obtained from the supplier without further purification. All melting points reported are uncorrected and were determined on a Stuart electric melting point apparatus. Elemental analysis were carried out in the Micro Analytical Center, Cairo University, Giza, Egypt. Thin-layer chromatography (TLC) was performed on Merk TLC aluminium sheets silica gel $60 \mathrm{~F}_{254}$ with detection by UV quenching at $254 \mathrm{~nm}$. IR spectra (in $\mathrm{KBr}, \mathrm{cm}^{-1}$ ) were recorded on FTIR 8201PC Shimadzu (Japan, 1995). ${ }^{1} \mathrm{H}$ NMR spectra were recorded on a Varian $300 \mathrm{MHz}$ (Germany, 1999) with residual proton signal of the deuterated solvent as the internal reference $\left(\delta_{\mathrm{H}}=7.26 \mathrm{ppm}\right.$ for $\mathrm{CDCl}_{3}$ and $\delta_{\mathrm{H}}=2.51 \mathrm{ppm}$ for DMSO-d6). TMS was used as an internal standard with chemical shifts $\delta$ in ppm from downfield to upfield. EIMS were recorded on a gas chromatographic GCMS -Qploopx Shimadzu (Japan, 1990).

\section{4-(3,4-Dichlorophenyl)-6-(furan-2-yl)pyrimidine-2-thiol (1)}

A mixture of 3,4-dichloroacetophenone $(1.89 \mathrm{~g}, 0.01 \mathrm{~mol})$, furfural $(0.96 \mathrm{~g}, 0.01 \mathrm{~mol})$, and thiourea $(0.76 \mathrm{~g}, 0.01 \mathrm{~mol})$ and $\mathrm{CH}_{3} \mathrm{ONa}(0.01 \mathrm{~mol})$ in $30 \mathrm{~mL} \mathrm{EtOH}$ was heated under reflux in an oil bath for $7 \mathrm{~h}$. The reaction mixture was cooled and the solid that obtained was filtered off, the filtrate was neutralized with $\mathrm{AcOH}$. The product that obtained was recrystallized from $\mathrm{PhCH}_{3}$ to give 1. Yield 56\%; mp 161-162 ${ }^{\circ} \mathrm{C}$; IR $\left(\mathrm{KBr}, \mathrm{cm}^{-1}\right)$ : $1451(\mathrm{C}=\mathrm{S}), 3059\left(\mathrm{CH}\right.$-arom.); ${ }^{1} \mathrm{H}-\mathrm{NMR}(300 \mathrm{MHz}$, DMSO- $d 6, \delta / \mathrm{ppm}): 3.82(\mathrm{~s}, 1 \mathrm{H}, \mathrm{SH}), 6.58\left(\mathrm{dd}, J_{1}=1.7 \mathrm{~Hz}, J_{2}=3.6 \mathrm{~Hz}, 1 \mathrm{H}\right.$, furyl-H), 7.11-7.18 (m, 3H, pyrimidine- $\mathrm{H} \&$ Ar- $\mathrm{H} \&$ furyl-H), 7.57-7.61 (m, 2H, Ar-H \& furyl-H), 7.89 (d, J=8.17Hz, $1 \mathrm{H}$, Ar-H). Anal. Calcd. for $\mathrm{C}_{14} \mathrm{H}_{8} \mathrm{Cl}_{2} \mathrm{~N}_{2} \mathrm{OS}(323.20): \mathrm{C}$ 52.03; H, 2.49; Cl, 21.94; N, 8.67; S, 9.92. Found: C, 52.33; H, 2.56; Cl, 22.36; N, 8.91; S, 9.70. MS: m/z 323[M] ${ }^{+}$, $325\left[\mathrm{M}^{+}+2\right]$, and $327\left[\mathrm{M}^{+}+4\right]$.

\section{4-(3,4-Dichlorophenyl)-6-(furan-2-yl)-2-hydrazinylpyrimidine (2)}

A solution of thioxopyrimidine $1(3.23 \mathrm{~g}, 0.01 \mathrm{~mol})$ and hydrazine hydrate $(0.75 \mathrm{~g}, 0.015 \mathrm{ml})$ in $20 \mathrm{~mL}$ EtOH was heated under reflux for $6 \mathrm{~h}$. The solid that formed after concentration and cooling was filtered off and crystallized from $\mathrm{PhH}$ to give 2. Yield $74 \%$; mp 129-131 ${ }^{\circ} \mathrm{C}$; IR $\left(\mathrm{KBr}, \mathrm{cm}^{-1}\right): 3071\left(\mathrm{CH}\right.$-arom.), 3190, $3327(\mathrm{NH}) ;{ }^{1} \mathrm{H}-\mathrm{NMR}(300 \mathrm{MHz}$, DMSO-d6, $\delta / \mathrm{ppm})$ : 4.86 (brs, $1 \mathrm{H}, \mathrm{NH}), 6.49$ (dd, $J_{1}=1.7 \mathrm{~Hz}, J_{2}=3.6 \mathrm{~Hz}, 1 \mathrm{H}$, furyl-H), $7.08(\mathrm{~s}, 1 \mathrm{H}$, pyrimidine- $\mathrm{H}), 7.17-7.24(\mathrm{~m}, 2 \mathrm{H}$, Ar- $\mathrm{H} \&$ furyl$\mathrm{H}), 7.49-7.67\left(\mathrm{~m}, 2 \mathrm{H}, \mathrm{Ar}-\mathrm{H}\right.$ \& furyl-H), $7.78(\mathrm{~d}, \mathrm{~J}=8.2 \mathrm{~Hz}, 1 \mathrm{H}, \mathrm{Ar}-\mathrm{H})$. Anal. Calcd. for $\mathrm{C}_{14} \mathrm{H}_{10} \mathrm{Cl}_{2} \mathrm{~N}_{4} \mathrm{O}(321.17): \mathrm{C}, 52.36 ; \mathrm{H}$, 3.14; Cl, 22.08; N, 17.45. Found: C, 52.62; H, 3.29; Cl, 21.87; N, 17.22. MS: m/z 321[M] $]^{+}, 323\left[\mathrm{M}^{+}+2\right], 325\left[\mathrm{M}^{+}+4\right]$.

\section{4-(3,4-Dichlorophenyl)-N-ethyl-6-(furan-2-yl)pyrimidin-2-amine (3)}

A mixture of thioxopyrimidine $1(3.23 \mathrm{~g}, 0.01 \mathrm{~mol})$ and $\mathrm{C}_{2} \mathrm{H}_{5} \mathrm{NH}_{2}(0.45 \mathrm{~g}, 0.01 \mathrm{ml})$ in $20 \mathrm{~mL}$ EtOH was heated under reflux for $6 \mathrm{~h}$. The solid that formed after concentration and cooling was filtered off and crystallized from $\mathrm{PhH}$ to give 3 . Yield 77\%; mp 142-143 ${ }^{\circ} \mathrm{C}$; IR (KBr, cm $\left.{ }^{-1}\right): 2928$ (CH-aliph.), 3064 (CH-arom.), $3176(\mathrm{NH}) ;{ }^{1} \mathrm{H}-\mathrm{NMR}(300 \mathrm{MHz}, \mathrm{DMSO}-d 6, \delta /$ ppm): $1.17\left(\mathrm{t}, J=7.1 \mathrm{~Hz}, 3 \mathrm{H}, \mathrm{CH}_{3}\right), 3.24\left(\mathrm{q}, J=7.1 \mathrm{~Hz}, 2 \mathrm{H}, \mathrm{CH}_{2}\right), 5.82$ (brs, $\left.1 \mathrm{H}, \mathrm{NH}\right), 6.56\left(\mathrm{dd}, J_{1}=1.7 \mathrm{~Hz}, J_{2}=3.6 \mathrm{~Hz}, 1 \mathrm{H}\right.$, furyl$\mathrm{H})$, 7.06-7.14 (m, 2H, Ar-H \& pyrimidine-H), 7.39-7.61 (m, 3H, Ar-H \& furyl-H), $7.83(\mathrm{~d}, 1 \mathrm{H}, \mathrm{J}=8.1 \mathrm{~Hz}$, Ar-H). Anal. Calcd. for $\mathrm{C}_{16} \mathrm{H}_{13} \mathrm{Cl}_{2} \mathrm{~N}_{3} \mathrm{O}$ (334.20): C, 57.50; H, 3.92; Cl, 21.22; N, 12.57. Found: $\mathrm{C}, 57.74 ; \mathrm{H}, 4.15 ; \mathrm{Cl}, 21.03 ; \mathrm{N}, 12.88$. 


\section{4-(3,4-Dichlorophenyl)-6-(furan-2-yl)-2-(piperidin-1-yl)pyrimidine (4)}

A mixture of thioxopyrimidine $1(3.23 \mathrm{~g}, 0.01 \mathrm{~mol})$ and piperidine $(0.85 \mathrm{~g}, 0.01 \mathrm{~mol})$ in $15 \mathrm{~mL} \mathrm{MeOH}$ was refluxed for $6 \mathrm{~h}$. The solid that formed after concentration and cooling was filtered off and crystallized from $\mathrm{AcOH}$ to give 4. Yield $65 \%$; $\mathrm{mp}$ $175^{\circ} \mathrm{C}$; IR (KBr, cm $\left.{ }^{-1}\right): 2941$ ( $\mathrm{CH}$-aliph.), 3059 (CH-arom.). Anal. Calcd. for $\mathrm{C}_{19} \mathrm{H}_{17} \mathrm{Cl}_{2} \mathrm{~N}_{3} \mathrm{O}$ (374.26): C, 60.97; $\mathrm{H}, 4.58 ; \mathrm{Cl}$, 18.95; N, 11.23. Found: C, 61.19; H, 4.35; Cl, 19.33; N, 11.62. MS: m/z 375[M] $]^{+}$377[ $\left.\mathrm{M}^{+}+2\right]$.

\section{2-(3,4-Dichlorophenyl)-4-(furan-2-yl)-6H-pyrimido[2,1-b]quinazolin-6-one (5)}

An equimolar amounts of thioxopyrimidine 1 (3.23 g, $0.01 \mathrm{~mol})$ and 2-aminobenzoic acid $(0.01 \mathrm{~mol})$ in 1,4-dioxane (30mL) was heated under reflux at $100^{\circ} \mathrm{C}$ for $2 \mathrm{~h}$. The reaction mixture was cooled down and a few drops of concentrated $\mathrm{H}_{2} \mathrm{SO}_{4}$ was added with stirring at $r$. $t$ and stirring was continued for $2 \mathrm{~h}$. Then the reaction mixture was poured over ice water and the solid that formed was collected and recrystallized from $\mathrm{PhH}$ to give 5 . Yield $53 \% ; \mathrm{mp} 120-122{ }^{\circ} \mathrm{C} ; \mathrm{IR}\left(\mathrm{KBr}, \mathrm{cm}^{-1}\right): 1676$ (CO), 3063 (CH-arom.); ${ }^{1} \mathrm{H}-\mathrm{NMR}$ (300 MHz, DMSO-d6, $\left.\delta / \mathrm{ppm}\right): 6.64-6.77$ (m, 2H, furyl-H), 7.43-7.56 (m, 4H, Ar-H \& furyl-H \& pyrimidine-H), 7.60-7.78 (m, 3H, Ar-H), 8.27-8.51 (m, $2 \mathrm{H}, \mathrm{Ar}-\mathrm{H})$. Anal. Calcd. for $\mathrm{C}_{21} \mathrm{H}_{11} \mathrm{Cl}_{2} \mathrm{~N}_{3} \mathrm{O}_{2}(408.24): \mathrm{C}$, 61.78; H, 2.72; Cl, 17.37; N, 10.29. Found: C, 62.14; H, 2.95; Cl, 17.12; N, 10.55.

\section{\{[4-(3,4-Dichlorophenyl)-6-(furan-2-yl)pyrimidin-2-yl]sulfanyl\}acetic acid (6)}

A mixture of compound 1 (3.23 g, $0.01 \mathrm{~mol})$ and chloroacetic acid $(0.95 \mathrm{~g}, 0.01 \mathrm{~mol})$, alcoholic $\mathrm{NaOH}(2 \mathrm{~mL}, 10 \%)$ in $30 \mathrm{~mL}$ $\mathrm{EtOH}$ was heated under reflux for $3 \mathrm{~h}$. The excess solvent was distiled off and the residue was poured up on water, neutralized with dil. $\mathrm{HCl}$. The solid that formed was washed with $\mathrm{H}_{2} \mathrm{O}$, filtered off and crystallized from AcOH to give 6 . Yield 59\%; mp 192-193 ${ }^{\circ} \mathrm{C}$; IR (KBr, cm $\left.{ }^{-1}\right)$ : 1154 (C-S), 1710 (CO), 3063 (CH-arom.), 3426 (OH). Anal. Calcd. for $\mathrm{C}_{16} \mathrm{H}_{10} \mathrm{Cl}_{2} \mathrm{~N}_{2} \mathrm{O}_{3} \mathrm{~S}$ (381.23): C, 50.41; H, 2.64; Cl, 18.60; N, 7.35; S, 8.41. Found: C, 50.76; H, 2.85; Cl, 18.83; N, 7.63; S, 8.17.

\section{Ethyl \{[4-(3,4-dichlorophenyl)-6-(furan-2-yl)pyrimidin-2-yl]sulfanyl\} acetate (7)}

A mixture of compound 1 (3.23 g, $0.01 \mathrm{~mol})$ and ethyl chloroacetate $(3.69 \mathrm{~g}, 0.03 \mathrm{~mol})$ in dry pyridine $(20 \mathrm{~mL})$ was heated under reflux for $4 \mathrm{~h}$. The reaction mixture after cooling was poured over $\mathrm{HCl} / \mathrm{crushed}$ ice. The solid obtained was filtered off and crystallized from AcOH to give 7. Yield 71\%; mp 137-139 ${ }^{\circ} \mathrm{C}$; IR ( $\left.\mathrm{KBr}, \mathrm{cm}^{-1}\right)$ : 1152(C-S), 1745 (CO), $3070(\mathrm{CH}$-arom.) ${ }^{1} \mathrm{H}-\mathrm{NMR}(300 \mathrm{MHz}, \mathrm{DMSO}-d 6, \delta / \mathrm{ppm}): 1.08$ (t, $\left.\mathrm{J}=6.9 \mathrm{~Hz}, 3 \mathrm{H}, \mathrm{CH}_{3}\right), 3.29\left(\mathrm{~s}, 2 \mathrm{H}, \mathrm{CH}_{2}\right), 3.93\left(\mathrm{q}, \mathrm{J}=7.9 \mathrm{~Hz}, 2 \mathrm{H}, \mathrm{CH}_{2}\right), 6.53$ (dd, $J_{1}=1.7 \mathrm{~Hz}, J_{2}=3.6 \mathrm{~Hz}, 1 \mathrm{H}$, furyl-H), 7.18-7.23 $(\mathrm{m}, 3 \mathrm{H}$, Ar- $\mathrm{H} \&$ pyrimidine-H\& furyl-H), 7.59-7.64 $(\mathrm{m}, 2 \mathrm{H}, \mathrm{Ar}-\mathrm{H} \&$ furyl-H), $7.88(\mathrm{~d}, 1 \mathrm{H}, \mathrm{J}=8.2 \mathrm{~Hz}, \mathrm{Ar}-\mathrm{H})$. Anal. Calcd. for $\mathrm{C}_{18} \mathrm{H}_{14} \mathrm{Cl}_{2} \mathrm{~N}_{2} \mathrm{O}_{3} \mathrm{~S}$ (409.29): C, 52.82; H, 3.45; Cl, 17.32; N, 6.84; S, 7.83. Found: $\mathrm{C}, 53.10 ; \mathrm{H}, 3.58 ; \mathrm{Cl}, 17.11 ; \mathrm{N}, 7.18 ; \mathrm{S}, 7.65$.

\section{2-\{[4-(3,4-Dichlorophenyl)-6-(furan-2-yl)pyrimidin-2-yl]sulfanyl\} acetohydrazide (8)}

A solution of thioxopyrimidine $7(4.09 \mathrm{~g}, 0.01 \mathrm{~mol})$ and hydrazine hydrate $(0.75 \mathrm{~g}, 0.015 \mathrm{~mol})$ in $20 \mathrm{~mL} \mathrm{EtOH}$ was heated under reflux for $8 \mathrm{~h}$. The solid that formed after concentration and cooling was filtered off and crystallized from $\mathrm{EtOH}$ to give 8. Yield 54\%; mp $171{ }^{\circ} \mathrm{C}$; IR $\left(\mathrm{KBr}, \mathrm{cm}^{-1}\right)$ : 1681 (CO), 2923 ( $\mathrm{CH}$-aliph.), 3151, $3390(\mathrm{NH})$. Anal. Calcd. for $\mathrm{C}_{16} \mathrm{H}_{12} \mathrm{Cl}_{2} \mathrm{~N}_{4} \mathrm{O}_{2} \mathrm{~S}$ (395.26): C, 48.62; H, 3.06; Cl, 17.94; N, 14.17; S, 8.11. Found: C, 48.94; H, 3.39; Cl, 18.26; N, 14.47; S, 8.32.

\section{Formation of $9 a, b$}

An equimolar mixture of compound 1 and 3-[(4-bromobenzoyl) and/or 2-furyl]prop-2-enoic acid (0.01 mol), and few drops of piperidine in $30 \mathrm{~mL}$ dry benzene was heated at $80^{\circ} \mathrm{C}$ in water bath for $3 \mathrm{~h}$. The excess solvent removed by steam distillation and the crude obtained was purified by recrystallization from $\mathrm{PhCH}_{3}$ to give $9 \mathrm{a}$ and $9 \mathrm{~b}$ respectively.

\section{5-(4-Bromo-phenyl)-2-[4-(3,4-dichloro-phenyl)-6-furan-2-yl-pyrimidin-2-ylsulfanyl]-5-}

oxo-pentanoic acid (9a): Yield $62 \%$; mp 220-222 ${ }^{\circ} \mathrm{C}$; IR (KBr, cm $\left.{ }^{-1}\right): 1710$ (CO), $2938(\mathrm{CH}$-aliph.), 3135,3300 $(\mathrm{OH}) ;{ }^{\mathrm{H}} \mathrm{HMR}(300 \mathrm{MHz}, \mathrm{DMSO}-d 6, \delta / \mathrm{ppm}): 2.39-2.43\left(\mathrm{~m}, 2 \mathrm{H}, \mathrm{CH}_{2}\right), 3.12\left(\mathrm{t}, J=7.1 \mathrm{~Hz}, 2 \mathrm{H}, \mathrm{CH}_{2}\right), 4.26(\mathrm{t}, J=7.8 \mathrm{~Hz}, 1 \mathrm{H}$, $\mathrm{CH}), 6.57\left(\mathrm{dd}, J_{1}=1.7 \mathrm{~Hz}, J_{2}=3.6 \mathrm{~Hz}, 1 \mathrm{H}\right.$, furyl-H), 7.16-7.23 $(\mathrm{m}, 3 \mathrm{H}$, Ar- $\mathrm{H} \&$ pyrimidine- $\mathrm{H} \&$ furyl-H), 7.60-7.82 $(\mathrm{m}, 7 \mathrm{H}, \mathrm{Ar}-\mathrm{H}$ \& furyl-H), 10.42 (brs, $1 \mathrm{H}, \mathrm{OH}$ ). Anal. Calcd. for $\mathrm{C}_{25} \mathrm{H}_{17} \mathrm{BrCl}_{2} \mathrm{~N}_{2} \mathrm{O}_{4} \mathrm{~S}$ (592.29): C, 50.70; H, 2.89; Cl, 11.97; N, 4.73; S, 5.41. Found: C, 50.97; H, 3.05; Cl, 11.64; N, 4.92; S, 5.66.

\section{2-\{[4-(3,4-Dichlorophenyl)-6-(furan-2-yl)pyrimidin-2-yl]sulfanyl\}-5-(furan-2-yl)-5-}

oxopentanoic acid (9b): Yield 69\%; mp 237-239 ${ }^{\circ} \mathrm{C}$; IR (KBr, $\left.\mathrm{cm}^{-1}\right): 1709,1715$ (CO), 2936 (CH-aliph.), 3345 (OH). Anal. Calcd. for $\mathrm{C}_{23} \mathrm{H}_{16} \mathrm{Cl}_{2} \mathrm{~N}_{2} \mathrm{O}_{5} \mathrm{~S}$ (503.36): C, 54.88; H, 3.20; Cl, 14.09; N, 5.57; S, 6.37. Found: C, 55.21; H, 3.43; Cl, $13.91 ; \mathrm{N}, 5.81 ; \mathrm{S}, 6.55$.

\section{7-(3,4-Dichlorophenyl)-5-(furan-2-yl)[1,2,4]triazolo[4,3-a]pyrimidine (10)}

A solution of compound $2(3.21 \mathrm{~g}, 0.01 \mathrm{~mol})$ in $10 \mathrm{~mL}$ freshly distilled $\mathrm{Ac}_{2} \mathrm{O}$ was heated under reflux for $1.5 \mathrm{~h}$. The solid that formed after concentration and cooling was filtered off and crystallized from $\mathrm{EtOH}$ to give 10 . Yield $76 \%$; $\mathrm{mp} 164-165{ }^{\circ} \mathrm{C}$ IR ( $\left.\mathrm{KBr}, \mathrm{cm}^{-1}\right)$ : 2955 (CH-aliph.), 3068 (CH-arom.); ${ }^{1} \mathrm{H}-\mathrm{NMR}(300 \mathrm{MHz}, \mathrm{DMSO}-\mathrm{d} 6, \delta / \mathrm{ppm}): 6.68$ (dd, $J_{1}=1.7 \mathrm{~Hz}, J_{2}=3.6 \mathrm{~Hz}$, $1 \mathrm{H}$, furyl-H), $7.13(\mathrm{~s}, 1 \mathrm{H}$, pyrimidine- $\mathrm{H}), 7.36(\mathrm{~d}, \mathrm{~J}=8.7 \mathrm{~Hz}, 1 \mathrm{H}, \operatorname{Ar}-\mathrm{H}), 7.83-8.03(\mathrm{~m}, 4 \mathrm{H}$, Ar- $\mathrm{H}$ \& furyl-H), $8.78(\mathrm{~s}, 1 \mathrm{H}$, triazole-H). Anal. Calcd. for $\mathrm{C}_{15} \mathrm{H}_{8} \mathrm{Cl}_{2} \mathrm{~N}_{4} \mathrm{O}$ (331.16): C, 54.40; $\mathrm{H}, 2.43 ; \mathrm{Cl}, 21.41 ; \mathrm{N}, 16.92$. Found: $\mathrm{C}, 54.69 ; \mathrm{H}, 2.62 ; \mathrm{Cl}$, $21.17 ; \mathrm{N}, 17.19$. 


\section{7-(3,4-Dichlorophenyl)-5-(furan-2-yl)[1,2,4]triazolo[4,3-a]pyrimidine-3(2H)-thione (11)}

To a solution of $2(3.21 \mathrm{~g}, 0.01 \mathrm{~mol})$ in dry pyridine $(30 \mathrm{~mL}), \mathrm{CS}_{2}(1.52 \mathrm{~g}, 0.02 \mathrm{~mol})$ was added and the reaction mixture was heated under reflux for $8 \mathrm{~h}$. After cooling the reaction mixture was poured onto ice $/ \mathrm{HCl}$ mixture and the solid that separated was washed with cold water, filtered off, and crystallized from EtOH to give 11. Yield $61 \% ; \mathrm{mp} 198{ }^{\circ} \mathrm{C}$; IR $(\mathrm{KBr}$, $\mathrm{cm}^{-1}$ ): $1453(\mathrm{C}=\mathrm{S}), 2977$ (CH-aliph.), $3400(\mathrm{NH})$. Anal. Calcd. for $\mathrm{C}_{15} \mathrm{H}_{8} \mathrm{Cl}_{2} \mathrm{~N}_{4} \mathrm{OS}$ (363.22): C, 49.60; $\mathrm{H}, 2.22 ; \mathrm{Cl}, 19.52 ; \mathrm{N}$, 15.43; S, 8.83. Found: C, 49.24; H, 2.38; Cl, 19.20; N, 15.74; S, 9.12. MS: m/z 363[M] ${ }^{+}, 365\left[\mathrm{M}^{+}+2\right], 367\left[\mathrm{M}^{+}+4\right]$.

\section{2-\{[4-(3,4-Dichlorophenyl)-6-(furan-2-yl)pyrimidin-2-yl]amino\}-1H-isoindole-1,3(2H)- dione (12)}

To a solution of $2(3.21 \mathrm{~g}, 0.01 \mathrm{~mol})$ in $n-\mathrm{BuOH}, 0.01 \mathrm{~mol}$ of phathalic acid anhydride was added. The reaction mixture was leaved overnight and the solid that formed was collected and crystallized from EtOAc to give 12. Yield $56 \% ; \mathrm{mp} 149-150$ ${ }^{\circ} \mathrm{C}$; IR $\left(\mathrm{KBr}, \mathrm{cm}^{-1}\right):$ 1685, $1737(\mathrm{CO}), 3118(\mathrm{NH}) ;{ }^{1} \mathrm{H}-\mathrm{NMR}(300 \mathrm{MHz}, \mathrm{DMSO}-\mathrm{d} 6, \delta / \mathrm{ppm}): 6.44(\mathrm{~d}, J=3.6 \mathrm{~Hz}, 1 \mathrm{H}$, furyl-H), $6.61\left(\mathrm{dd}, J_{1}=1.7 \mathrm{~Hz}, J_{2}=3.6 \mathrm{~Hz}, 1 \mathrm{H}\right.$, furyl-H), 7.14-7.20 (m, $2 \mathrm{H}$, Ar- $\mathrm{H}$ \& pyrimidine- $\left.\mathrm{H}\right), 7.54-7.81(\mathrm{~m}, 3 \mathrm{H}$, Ar- $\mathrm{H}$ \& furyl-H), 7.93-8.04 (m, 4H, Ar-H), 8.24 (brs, $1 \mathrm{H}, \mathrm{NH}$ ). Anal. Calcd. for $\mathrm{C}_{22} \mathrm{H}_{12} \mathrm{Cl}_{2} \mathrm{~N}_{4} \mathrm{O}_{3}$ (451.26): $\mathrm{C}, 58.55 ; \mathrm{H}, 2.68 ; \mathrm{Cl}, 15.71 ; \mathrm{N}$, 12.42. Found: C, 58.85; H, 2.86; Cl, 15.90; N, 12.75.

\section{4-(3,4-Dichlorophenyl)-2-(3,5-dimethyl-1 H-pyrazol-1-yl)-6-(furan-2-yl)pyrimidine (13)}

A mixture of $2(3.21 \mathrm{~g}, 0.01 \mathrm{~mol})$, acetyl acetone $(0.54 \mathrm{~g}, 0.01 \mathrm{~mol})$, and few drops of piperidine in $30 \mathrm{~mL} \mathrm{EtOH}$ was heated at $70^{\circ} \mathrm{C}$ for $5 \mathrm{~h}$. The solid that separated after concentration and cooling was filtered off and recrystallized from light pet. ether $\left(80-100^{\circ}\right)$ to give 13. Yield 49\%; mp 138-140 ${ }^{\circ} \mathrm{C}$; IR $\left(\mathrm{KBr}, \mathrm{cm}^{-1}\right): 1610(\mathrm{C}=\mathrm{N}), 2911(\mathrm{CH}-$ aliph. $), 3048(\mathrm{CH}-$ arom.). Anal. Calcd. for $\mathrm{C}_{19} \mathrm{H}_{14} \mathrm{Cl}_{2} \mathrm{~N}_{4} \mathrm{O}$ (385.25): C, 59.24; $\mathrm{H}, 3.66 ; \mathrm{Cl}, 18.41 ; \mathrm{N}, 14.54$. Found: $\mathrm{C}, 59.03 ; \mathrm{H}, 3.79 ; \mathrm{Cl}$, 17.94; N, 14.82. MS: $\mathrm{m} / \mathrm{z} 385\left[\mathrm{M}^{+}, 387\left[\mathrm{M}^{+}+2\right]\right.$

\section{4-[\{2-[4-(3,4-Dichlorophenyl)-6-(furan-2-yl)pyrimidin-2-yl] hydrazinylidene\}methyl]phenol (14)}

A mixture of $2(3.21 \mathrm{~g}, 0.01 \mathrm{~mol})$, 4-hydroxybenzaldehyde (1.22 g, $0.01 \mathrm{~mol})$, in 1,4-dioxane $(20 \mathrm{~mL})$ was refluxed for $5 \mathrm{~h}$. The solid that separated after concentration and cooling was recrystallized from EtOH to give 14. Yield 82\%; mp 176-177 ${ }^{\circ} \mathrm{C}$; IR $\left(\mathrm{KBr}, \mathrm{cm}^{-1}\right): 1600(\mathrm{C}=\mathrm{N}), 3290(\mathrm{NH}), 3480(\mathrm{OH}) ;{ }^{1} \mathrm{H}-\mathrm{NMR}(300 \mathrm{MHz}, \mathrm{DMSO}-\mathrm{d} 6, \delta / \mathrm{ppm}): 6.67-6.69(\mathrm{~m}, 4 \mathrm{H}, \mathrm{Ar}-\mathrm{H}$ \& $=\mathrm{CH}$ \& furyl-H), 6.84-6.87 (m, $5 \mathrm{H}, \mathrm{Ar}-\mathrm{H}$ \& furyl- $\mathrm{H}$ \& pyrimidine- $\mathrm{H}), 8.30-8.54(\mathrm{~m}, 3 \mathrm{H}, \mathrm{ArH}), 10.03\left(\mathrm{brs}, 1 \mathrm{H}, \mathrm{NH}, \mathrm{D} \mathrm{O}^{\mathrm{O}}\right.$ exchangable), 10.09 (brs, $1 \mathrm{H}, \mathrm{OH}, \mathrm{D}_{2} \mathrm{O}$ exchangable). Anal. Calcd. for $\mathrm{C}_{21} \mathrm{H}_{14} \mathrm{Cl}_{2} \mathrm{~N}_{4} \mathrm{O}_{2}$ (425.27): $\mathrm{C}, 59.31 ; \mathrm{H}, 3.32 ; \mathrm{Cl}$, 16.67; N, 13.17. Found: C, 59.04; H, 3.14; Cl, 16.21; N, 13.38. MS: m/z 425[M] .

\section{2-[4-(3,4-Dichlorophenyl)-6-(furan-2-yl)pyrimidin-2-yl]-N-phenylhydrazine carboxamide (15)}

A mixture of compound $2(3.21 \mathrm{~g}, 0.01 \mathrm{~mol})$, phenyl isocyanate $(0.01 \mathrm{~mol})$ and a catalytic amount of $\mathrm{Et}_{3} \mathrm{NH}_{2}$ in $\mathrm{MeOH}(20$ $\mathrm{mL}$ ) was refluxed for $7 \mathrm{~h}$. The reaction mixture was concentrated and the precipitate formed was filtered off and recrystallized from $\mathrm{PhH}$ to give 15. Yield 73\%; mp 112-114 ${ }^{\circ} \mathrm{C}$; IR $\left(\mathrm{KBr}, \mathrm{cm}^{-1}\right): 1605(\mathrm{C}=\mathrm{N}), 1709(\mathrm{CO}), 3136,3300(\mathrm{NH})$; ${ }^{1} \mathrm{H}-\mathrm{NMR}\left(300 \mathrm{MHz}, \mathrm{CDCl}_{3}, \delta / \mathrm{ppm}\right): 6.95-6.99(\mathrm{~m}, 3 \mathrm{H}, \mathrm{Ar}-\mathrm{H}$ \& furyl-H), 7.24-7.29 (m, 5H, Ar-H \& furyl- $\mathrm{H}), 7.47-7.50(\mathrm{~m}$, $4 \mathrm{H}$, Ar- $\mathrm{H}$ \& pyrimidine-H), 9.64 (brs, $3 \mathrm{H}, 3 \mathrm{NH}, \mathrm{D}_{2} \mathrm{O}$ exchangable). Anal. Calcd. for $\mathrm{C}_{21} \mathrm{H}_{15} \mathrm{Cl}_{2} \mathrm{~N}_{5} \mathrm{O}_{2}(440.28): \mathrm{C}, 57.29 ; \mathrm{H}$, 3.43; Cl, 16.10; N, 15.91. Found: C, 57.08; H, 3.25; Cl, 16.43; N, 16.22.

\section{$N^{\prime}$-[(4-Chlorophenyl)methylidene]-2-\{[4-(3,4-dichlorophenyl)-6-(furan-2-yl)pyrimidin-2- yl]sulfanyl\}acetohydrazide (16)}

An equimolar ratio of $2(3.21 \mathrm{~g}, 0.01 \mathrm{~mol})$, 4-chlorobenzaldehyde $(1.41 \mathrm{~g}, 0.01 \mathrm{~mol})$, in $\mathrm{EtOH}(20 \mathrm{~mL})$ was refluxed for $6 \mathrm{~h}$. The solid that separated after concentration and cooling was recrystallized from EtOH to give 16. Yield 77\%; $\mathrm{mp} 211-213$ ${ }^{\circ} \mathrm{C}$; IR ( $\left.\mathrm{KBr}, \mathrm{cm}^{-1}\right)$ : $1682(\mathrm{CO}), 2934$ (CH-aliph.), $3300(\mathrm{NH})$. Anal. Calcd. for $\mathrm{C}_{23} \mathrm{H}_{15} \mathrm{Cl}_{3} \mathrm{~N}_{4} \mathrm{O}_{2} \mathrm{~S}(517.82): \mathrm{C}, 53.35 ; \mathrm{H}, 2.92$; Cl, 20.54; N, 10.82; S, 6.19. Found: C, 53.67; H, 2.75; Cl, 20.28; N, 11.16; S, 6.53. MS: m/z 518[M] ${ }^{+}$.

\section{4-(3,4-Dichloro-phenyl)-6-furan-2-yl-2-(5-p-tolyl-2,3-dihydro-[1,3,4]oxadiazol-2-yl methylsulfanyl)-pyrimidine (17)}

To a mixture of 8 (3.95 g, $0.01 \mathrm{~mol})$ and P-toluic acid $(1.36 \mathrm{~g}, 0.01 \mathrm{~mol})$ in $20 \mathrm{~mL}$ 1,4-dioxane, $10 \mathrm{~mL}$ of $\mathrm{POCl}_{3}$ was added portion wise with stirring at $0{ }^{\circ} \mathrm{C}$. The temperature was elevated gradually to $120^{\circ} \mathrm{C}$ and kept for $1 \mathrm{~h}$. After cooling the reaction mixture was poured onto ice/water mixture and the solid that separated was filtered off and crystallized from EtOH to give 17. Yield $58 \%$; mp 185-186 ${ }^{\circ} \mathrm{C}$; IR $\left(\mathrm{KBr}, \mathrm{cm}^{-1}\right): 1620(\mathrm{C}=\mathrm{N}), 2986\left(\mathrm{CH}\right.$-aliph.), $3268(\mathrm{NH}) ;{ }^{1} \mathrm{H}-\mathrm{NMR}(300$ $\mathrm{MHz}$, DMSO-d6, $\delta / \mathrm{ppm}): 2.13\left(\mathrm{~s}, 3 \mathrm{H}, \mathrm{CH}_{3}\right), 3.54\left(\mathrm{~s}, 2 \mathrm{H}, \mathrm{CH}_{2}\right), 6.54(\mathrm{~d}, \mathrm{~J}=3.8 \mathrm{~Hz}, 1 \mathrm{H}$, furyl-H), 7.19-7.24 (m, $4 \mathrm{H}, \mathrm{Ar}-\mathrm{H}$, pyrimidine-H \& furyl-H), 7.58-7.65 (m, $4 \mathrm{H}$, Ar- $\mathrm{H}$ \& furyl-H), 7.86-8.09 (m, 2H, Ar-H). Anal. Calcd. for $\mathrm{C}_{24} \mathrm{H}_{16} \mathrm{Cl}_{2} \mathrm{~N}_{4} \mathrm{O}_{2} \mathrm{~S}$ (495.38): C, 58.19; H, 3.26; Cl, 14.31; N, 11.31; S, 6.47. Found: C, 58.38; H, 3.43; Cl, 13.89; N, 10.93; S, 6.09. 


\section{Formation of 18 and/or 19}

A mixture of $8(3.95 \mathrm{~g}, 0.01 \mathrm{~mol})$, ethyl acetoacetate $(0.70 \mathrm{~g}, 0.01 \mathrm{~mol})$ and/or acetyl acetone $(0.54 \mathrm{~g}, 0.01 \mathrm{~mol})$, and few drops of piperidine in $30 \mathrm{~mL} \mathrm{EtOH}$ was heated at $70{ }^{\circ} \mathrm{C}$ for $6 \mathrm{~h}$. The solid that separated after concentration and cooling was filtered off and recrystallized from AcOH to give $2.27 \mathrm{~g} \mathrm{(49 \% )} \mathrm{of} 18$ and $2.64 \mathrm{~g} \mathrm{(57 \% )} \mathrm{of} 19$.

\section{2-(\{[4-(3,4-Dichlorophenyl)-6-(furan-2-yl)pyrimidin-2-yl]sulfanyl\}acetyl)-5-methyl-2,4-} dihydro-3H-pyrazol-3-one (18): Yield 49\%; mp 178-180 ${ }^{\circ} \mathrm{C}$; $\mathrm{IR}\left(\mathrm{KBr}, \mathrm{cm}^{-1}\right): 1611(\mathrm{C}=\mathrm{N}), 1665(\mathrm{CO}), 2938$ (CH-aliph.); ${ }^{1} \mathrm{H}-\mathrm{NMR}(300 \mathrm{MHz}, \mathrm{DMSO}-d 6, \delta / \mathrm{ppm}): 1.89\left(3 \mathrm{H}, s, \mathrm{CH}_{3}\right), 3.40\left(2 \mathrm{H}, s, \mathrm{CH}_{2}\right), 3.55\left(2 \mathrm{H}, s, \mathrm{CH}_{2}\right), 6.53(1 \mathrm{H}, d d$, $J_{1}=1.7, J_{2}=3.6$, furyl-H), $7.03(1 \mathrm{H}, s$, pyrimidine-H), 7.20-7.26 $(2 \mathrm{H}, m$, Ar- $\mathrm{H} \&$ furyl-H), 7.59-7.76 $(3 \mathrm{H}, \mathrm{m}, \mathrm{Ar}-\mathrm{H} \&$ furyl-H). Anal. Calcd. for $\mathrm{C}_{20} \mathrm{H}_{14} \mathrm{Cl}_{2} \mathrm{~N}_{4} \mathrm{O}_{3} \mathrm{~S}$ (461.32): C, 52.07; H, 3.06; Cl, 15.37; N, 12.14; S, 6.95. Found: C, 52.32; H, 3.27; Cl, $15.72 ; \mathrm{N}, 12.35 ; \mathrm{S}, 7.23$.

\section{2-\{[4-(3,4-Dichlorophenyl)-6-(furan-2-yl)pyrimidin-2-yl]sulfanyl\}-1-(3,5-dimethyl}

pyrazolidin-1-yl)ethanone (19): Yield 57\%; mp 142-144 ${ }^{\circ} \mathrm{C}$; IR (KBr, cm $\left.{ }^{-1}\right): 1679$ (CO), 2946 (CH-aliph.). Anal. Calcd. for $\mathrm{C}_{21} \mathrm{H}_{20} \mathrm{Cl}_{2} \mathrm{~N}_{4} \mathrm{O}_{2} \mathrm{~S}$ (463.38): C, 54.43; H, 4.35; Cl, 15.30; N, 12.09; S, 6.92. Found: C, 54.14; H, 4.52; Cl, 15.03; N, 12.36; S, 7.18 .

\section{RESULTS AND DISCUSSION}

The titled compound 4-(3,4-dichlorophenyl)-6-(furan-2-yl)pyrimidine-2-thiol (1) was prepared via base catalysed condensation of 3,4-dichloroacetophenone, furfural, and thiourea (one-pot, three components synthesis) or by condensation of furfurylidene-3,4-dichloroacetophenone with thiourea in the presence of MeONa as a catalyst. The reaction possibly takes place via Aza-Michael addition to the unsaturated carbonyl moiety followed by cyclocondensation reaction to give the desired thioxopyrimidine 1 . The reaction possibly takes place via the following mechanism, (Scheme 1).

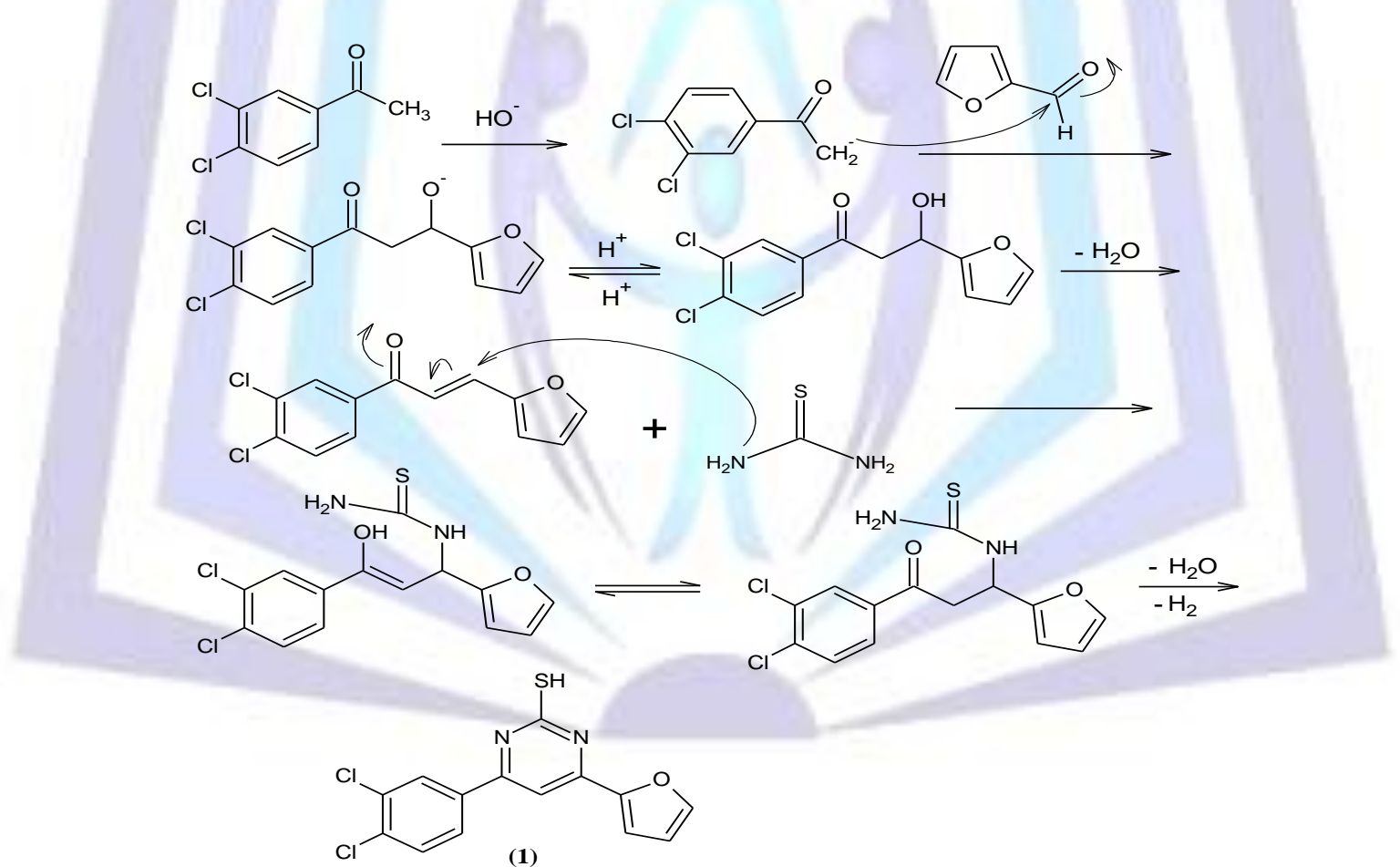

Scheme 1. Reaction mechanism for compound 1 formation.

The behaviour of thioxopyrimidine 1 towards nitrogen nucleophiles has been studied; its reaction with hydrazine hydrate was chosen as a model reaction, where the interaction of compound 1 with hydrazine hydrate in EtOH afforded 2 (Scheme 2). The reaction takes place via nucleophilic attack at the electrophilic center at carbon of $\mathrm{C}=\mathrm{S}$ group followed by loss of $\mathrm{H}_{2} \mathrm{~S}$ molecule and aromatization to the desired product. When thioxopyrimidine 1 was submitted to react with ethylamine yielded 2-ethylaminopyrimidine 3. Condensation of thioxopyrimidine derivative 1 with piperidine in $\mathrm{MeOH}$ gave 2 piperidinopyrimidine 4. Moreover, interaction of thioxopyrimidine 1 with 2-aminobenzoic acid has been investigated to proceed via refluxing a mixture of them in 1,4-dioxane followed by treatment with few drops of concentrated $\mathrm{H}_{2} \mathrm{SO}_{4}$ with continuous stirring at room temperature and resulted in the fused pyrimidino[2,3-b]quinazolinone $\mathbf{5}$ (Scheme 2). The IR spectrum of 5 revealed strong absorption band at $1660 \mathrm{~cm}^{-1}$ due to $v_{\mathrm{C}=\mathrm{O}}$ and devoid any band for $v_{\mathrm{C}=\mathrm{S}}$ and $v_{\mathrm{NH}}$. The reaction possibly takes place via nucleophilic attack of amino group on electrophilic center $(C=S)$ followed by acid catalysed dehydration to give the desired product. 
<smiles>Cc1cc(N)nc(SCC(=O)NN)n1</smiles>

(8)<smiles>CCOC(=O)CSc1nc(Br)cc(Br)n1</smiles>

(7)<smiles>[R]CCCCCCCC(Sc1nc(Br)cc(Br)n1)C(=O)O</smiles>

$$
\mathrm{b}, \mathrm{R}=\mathrm{C}_{4} \mathrm{H}_{3} \mathrm{O} \text { (2) }
$$

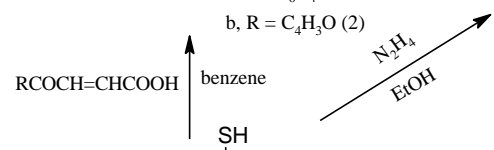<smiles>[Al+2]</smiles>

(2)

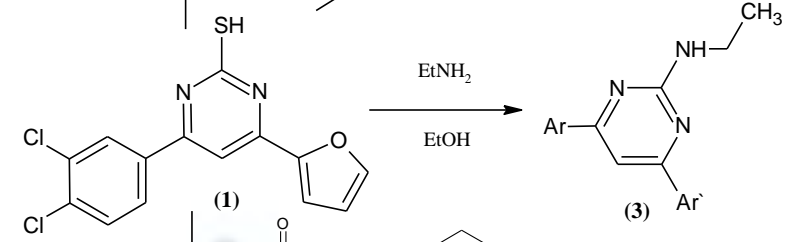<smiles></smiles>

(4)

(5)

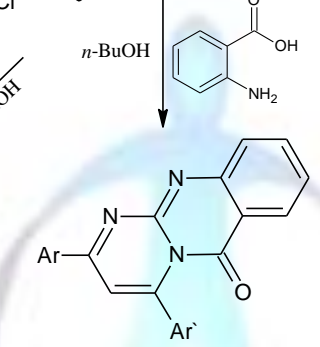

$\mathrm{Ar}^{`}=\mathrm{C}_{4} \mathrm{H}_{3} \mathrm{O}(2)$

$$
\text { Ar }=\mathrm{C}_{4} \mathrm{H}_{3} \mathrm{O}(2)
$$

$\mathrm{Ar}=\mathrm{C}_{6} \mathrm{H}_{3} \cdot \mathrm{Cl}_{2}(3,4)$

Scheme 2. Behaviour of thioxopyrimidine 1 towards nitrogen and carbon nucleophiles.

On the other hand, an understanding of the chemical behavior of thiopyrimidone $\mathbf{1}$ is of major importance in order to design the key starting material for production of many heterocyclic compounds with pharmecutical applications. The aim of this work is to determine the structures and mechanisms of the products obtained from the interaction of the thiopyrimidone with different carbon electrophiles.

In all solvents, except petrol at high dilution, the 2- and 4-hydroxypyridines are extremely unfavoured in lactam-lactim dynamic equilibrium establishment [24]. Polar solvation effects strongly and favour the amide-like structure. In case of 2 thioxopyrimidine, the strong $\pi$-back bonding in the $\mathrm{SH}$ group perhaps it can be used to explain why some properties of thiol compounds are rather different than phenolic compounds. In this context, thioxopyrimidine 1 was allowed to react with chloroacetic acid in alcoholic $\mathrm{NaOH}$ and the 2-carboxythiomethoxy-pyrimidine 6 was furnished (Scheme 2). Solubility of compound $\mathbf{6}$ in aqueous solution of sodium bicarbonate is a good evidence for its formation. The reaction possibly takes place via nucleophilic attack of sulphur anion on the alkyl moiety of chloroacetic acid via $\mathrm{S}_{\mathrm{N}} 2$ mechanism, this presented as follows (Scheme 3).<smiles>Cc1cc(Br)nc(S)n1</smiles><smiles>O=C(O)CSc1nc(Br)cc(Br)n1</smiles>

(6)

Scheme 3. Mechanism for compound 6 formation. 
Here the author offers a speculation to explain the activities of the thioamide and iminothiol equilibrium based on their thermodynamic and kinetic control under the experimental conditions. Firstly, in the presence of alcoholic $\mathrm{NaOH}$ the conjugate base of the iminothiol tautomer is more thermodynamically stable than conjugate base derived from thioamide tautomer via the $\pi$-back donation involving the vacant $d$-orbital of the sulphur atom. Thereafter, the iminothiol tautomer is more predominate under such condition. Secondly, sulphur anion is strong nucleophilic than nitrogen anion (nucleophilicity is kinetic control). Thus, the iminothiol tautomer is more thermodynamically and kinetically favoured than thioamide tautomer, which practically spells out the reactivity of the iminothiol tautomer.

Similarly, when 1 was submitted to react with ethyl chloroacetate in warming pyridine afforded 2 ethoxycarbonylthiomethoxy-pyrimidine $\mathbf{7}$. The structure of compound $\mathbf{7}$ was proved chemically via its reaction with hydrazine hydrate and the 2-hydrazinocarbonylthiomethoxypyrimidine derivative 8 was obtained (Scheme 2). The reaction possibly takes place via tetrahedral mechanism involving lone pair of nitrogen and acyl moiety of ester group. In the present work, we wish to point out the interaction of thiopyrimidone derivative 1 with $\beta$-(4-bromobenzoyl and/or 2 furyl)acrylic acid in the presence of piperidine as catalyst, The thia Michael adducts $9 \mathbf{a}, \mathbf{b}$ were obtained (Scheme 2).

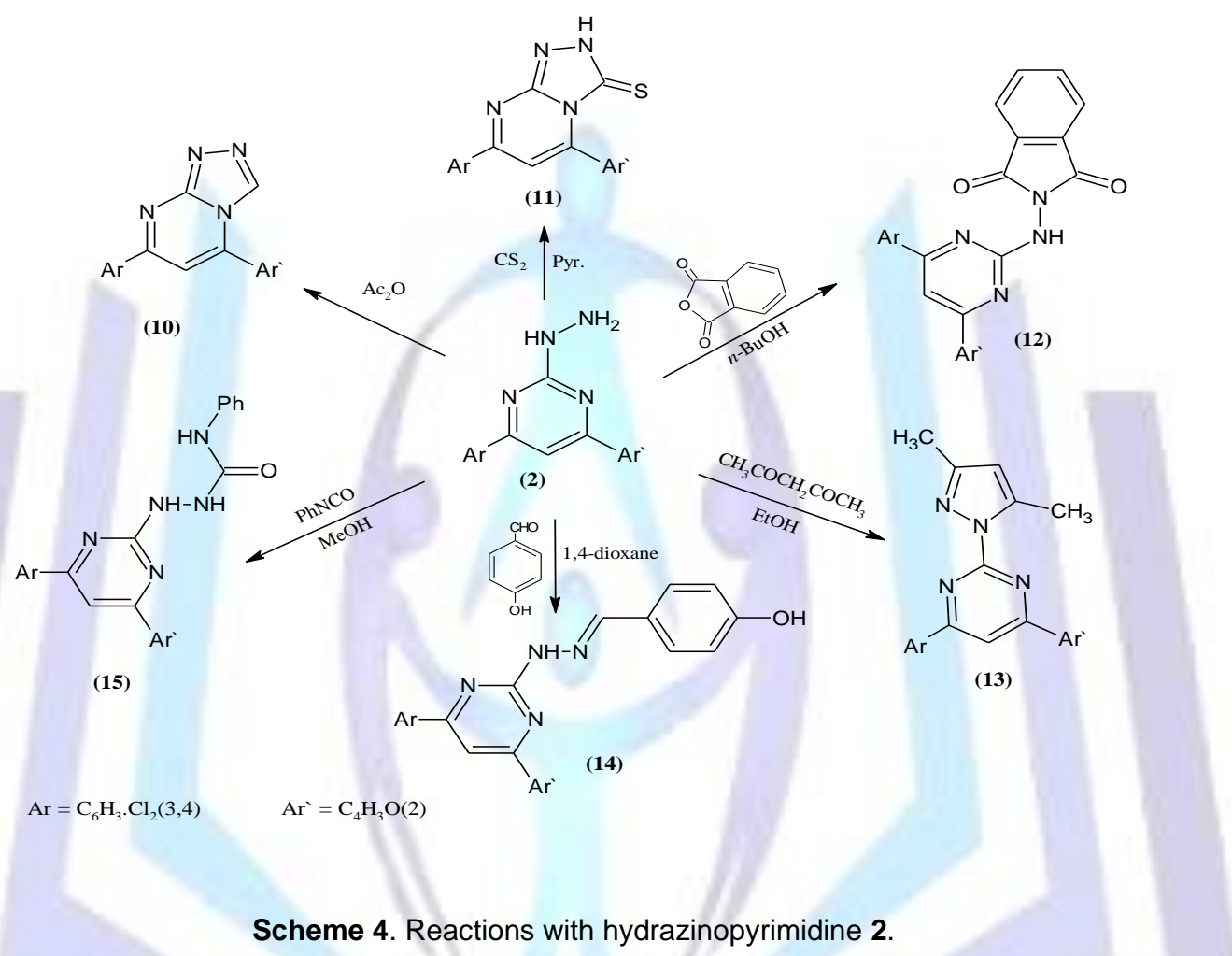

The chemistry of pyrimidines and thiopyrimidines have become in the last few years a subject of great interest, because they display some interesting properties in the field of organic chemistry [24,25]. In the present study, the authors have successful attempts to prepare hydrazinopyrimidine 2 as key starting material for synthesis some interesting nitrogen bridgehead compounds. Indeed, the hydrazino derivative 2 was allowed to react with $\mathrm{Ac}_{2} \mathrm{O}$ and the 2-methyl-1,3,4triazolo[1,5-b]pyrimidine 10 was afforded. Moreover, the hydrazino derivative 2 was submitted to react with $\mathrm{CS}_{2}$ in pyridine and resulted in 2-thioxo-1,3,4-trizolo[1,5-b]pyrimidine 11 (Scheme 4). Recently [26], it was reported that acid anhydrides reacted with 3 -aminoquinazolinone derivatives and gave phthalimid $4(3 H)$-quinazolinone. In the present work we wish to point out the reaction of hydrazino derivative 2 with phthalic acid anhydride. Thus, when compound 2 was interacted with phthalic acid anhydride in an oil bath it yielded 2-phthalimidoamino-4-(2-furyl)-6-(3,4-dichlorophenyl)pyrimidine 12 (Scheme 4). The reaction takes place via acylation of hydrazino moiety by phthalic acid anhydride followed by ring closure to give the desired product.

More recently [27], it was reported that, hydrazide derivatives condensed with acetylacetone in boiling EtOH and yielded the corresponding pyrazole derivative. In the present work the author thought to investigate the behavior of the hydrazinopyrimidine derivative towards acetylacetone. Indeed, compound $\mathbf{2}$ was allowed to react with acetylacetone and the pyrimidine 13 was obtained (Scheme 4).

Moreover, 2 was allowed to react with 4-hydroxybenzaldehyde in 1,4-dioxane and yielded the corresponding hydrazone 14 (Scheme 4). The reaction takes place via nucleophilic attack of lone pair of nitrogen on the carbonyl group followed by dehydration to give the more thermodynamically hydrazone derivative. It is well known that the hydrazide moiety in pyridone derivative interacted with phenyl isothiocynate and gave hydrazinocarbothioamide derivatives [27]. This applies fully to hydrazino derivative 2 and yielded 2-phenylthiocarbamoylhydrazinopyrimidine 15 (Scheme 4). 


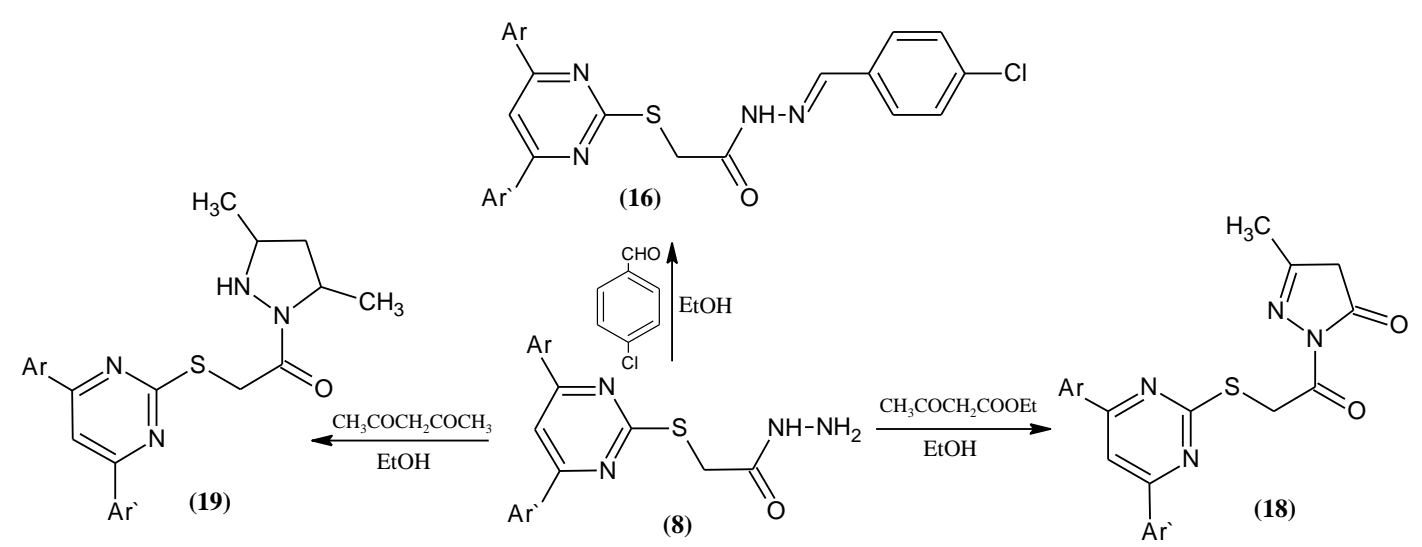<smiles>Cc1ccc(-c2nnc(CSc3nc(Br)cc(Br)n3)o2)cc1</smiles>

Scheme 5. Reactions with hydrazinocarbonylthiomethoxypyrimidine 8.

On the other hand, interaction of the aminocarbamoyl derivative 8 with 4-chlorobenzaldehyde in EtOH afforded the corresponding hydrazone 16. The reaction possibly takes place via nucleophilic attack of lone pair of nitrogen on electrophilic carbon of aldehyde followed by lose of water molecule to give the more thermodynamically stable adduct $\mathbf{1 6}$. Interaction of $P$-toluic acid with 8 in the presence of $\mathrm{POCl}_{3}$ yielded the 2-[2-(4-methylphenyl)-1,3,4-0xadiazol-5yl]thiomethoxy-4-(2-furyl)-6-(3,4-dichlorophenyl)pyrimidine (17) (Scheme 5).

When the hydrazide 8 was submitted to react with ethyl acetoacetate afforded 2-(3-methyl-5-oxo-pyrazolin-1$\mathrm{yl}$ )carbonylthiomethoxy-pyrimidine 18. The reaction takes place via interaction of lone pair of nitrogen atom with carbonyl moiety of ester [via nucleophilic addition to carbonyl group] followed by ring closure to give the desired product $\mathbf{1 8}$. The hydrazide derivative 8 interacted with acetylacetone yielded 2-[(3,5-dimethylpyrazol-1-yl)]carbonylthiomethoxy-pyrimidine 19 (Scheme 5).

\section{CONCLUSION}

In summary, pyrimidine-2-thiol (1) has been successfully synthesized and utilized as building block of some heterocyclic systems as well as a novel series of thioxopyrimidine derivatives in excellent yield.

\section{REFERENCES}

[1] P. Biginelli, Gazz. Chim. Ital., 23, 360 (1893).

[2] B. Schnell, W. Krenn, K. Faber, C. O. Kappe, J. Chem. Soc., Perkin Trans 1, 4382(2000).

[3] S.I. Filimonov, Z. V. Chirkova, I. G. Abramov, S. T. Firgang, G. A. Stashina, K. Y. Suponitsky, Mendeleev Communications, 21(1), 46(2011).

[4] S. S. Bahekar, D. B. Shinde, Acta Pharm., 53, 223(2003).

[5] C. O. Kappe, Eur. J. Med. Chem., 35, 1043(2000).

[6] S. F. Mohamed, E. M. Flefel, A. E. Amr, D. N. El-Shafy, Eur. J. Med. Chem., 45(4), 1494(2010).

[7] M. Modica, M. Santagati, A. Santagati, V. Cutuli, N. Mangano, A. Caruso, Pharmazie, 55, 500(2000).

[8] B. Tozkoparan, M. Ertan, P. Kelicen, R. Demirdamar, Farmaco, 54, 588(1999).

[9] Y. K. Gupta, V. Gupta, S. Singh, J. Pharm. Res., 7(6), 491-495(2013).

[10] M. I. Al-Ashmawy, S. A. El-Feky, Z. K. El-Samii, N. A. Osman, Boll. Chim. Farm., 136, 492(1997).

[11] S. K. Sharma, P. Kumar, B. Narasimhan, K. Ramasamy, V. Mani, R. K. Mishra, A. Abdul Majeed, Eur. J. Med. Chem., 48, 16(2012). 
[12] R. Kumar, M. Nath, D. L. Tyrrell, J. Med. Chem., 45, 2032(2002).

[13] E. Gossnitzer, G. Feierl, U. Wagner, Eur. J. Pharm. Sci., 15, 49(2002).

[14] D. B. Shinde, N. D. Shinde, M. S. Shingare, Indian J. Chem., 34B, 920(1995).

[15] Rovnyak, K. S. Atwal, A. Hedberg, S. D. Kimball, S. Moreland, J. Z. Cougoutas, B. C. O`Reilly, J. Schwartz, M. F. Malley, J. Med. Chem., 35, 3254(1992).

[16] G. J.Grover, S. Dzwonczyk, D. M. McMullen, D. E. Normandin, C. S. Parham, P. G. Sleph, S. Moreland, J. Cardiovasc. Pharm., 26, 289(1995).

[17] J. C. Barrow, P. G. Nantermet, H. G. Selnick, K. L. Glass, K. E. Rittle, K. F. Gilbert, T. G. Steele, C. F. Homnick, R. M. Freidinger, R. W. Ransom, P. Kling, D. Reiss, T. P. Broten, T. W. Schorn, R. S. L. Chang, S. S. O Malley, T. V. Olah, J. D. Ellis, A. Barrish, K. Kassahun, P. Leppert, D. Nagarathnam, C. Forray, J. Med. Chem., 43, 2703(2000).

[18] T. U. Mayer, T. M. Kapoor, S. J. Haggarty, S. L. King, M. T. J. Schreiber, Science, 286, 971(1999).

[19] S. J. Haggarty, T. U. Mayer, D. T. Miyamoto, R. Fathi, R. W. King, T. J. Mitchison, S. L. Schreiber, Chem. Biol., 7 , 275(2000).

[20] K. S. Atwal, G. C. Rovnyak, S. D. Kimball, D. M. Floyd, S. Moreland, B. N. Swanson, J. Z. Gougoutas, J. Schwartz, K. M. Smillie, M. F. Malley, J. Med. Chem., 33, 2629(1990).

[21] Rovnyak, S. D. Kimball, B. Beyer, G. Cucinotta, J. D. DiMarco, J. Gougoutas, A. Hedberg, M. Malley, J. P. McCarthy, R. Zhang, S. Moreland, J. Med. Chem., 38, 119(1995).

[22] L. Heys, C. G. Moore, P. Murphy, J. Chem. Soc. Rev., 29, 57(2000).

[23] A. D. Patil, N. V. Kumar, W. C. Kokke, M. F. Bean, A. J. Freyer, C. De Brosse, S. Mai, A. Truneh, D. J. Faulkner, B. Carte, A. L. Breen, R. P. Hertzberg, R. K. Johnson, J. W. Westley, B. C. M. Potts, J. Org. Chem., 60, 1182(1995).

[24] E. E. Flefel, M.A. Salama, M. El-Shahat, M. A. El-Hashash, A. F. El-Farargy, Phosphorus, Sulfur, Silicon and Related Elements, 182(8), 1739-1756(2007).

[25] M. A. Raj, S. B. Revin, S. A. John, Bioelectrochemistry, 89, 1-10(2013).

[26] F. R. Mohammed, H. M. Bakeer, M. A. El-Hashash, Asian J. Chem., 21(7), 5004-5014(2009).

[27] E. R. Kotb, M. A. El-Hashash, M. A. Salama, H. S. Kalf, N. A. M. Abdel Wahed, Acta Chim. Slov., 56, 908-919(2009). 